\title{
Geometric cluster Monte Carlo simulation
}

\author{
J. R. Heringa ${ }^{1}$ and H. W. J. Blöte ${ }^{1,2}$ \\ ${ }^{1}$ Faculty of Applied Physics, Delft University of Technology, P.O. Box 5046, 2600 GA Delft, The Netherlands \\ ${ }^{2}$ Lorentz Institute, Leiden University, P.O. Box 9506, 2300 RA Leiden, The Netherlands
}

(Received 22 October 1997)

\begin{abstract}
We discuss a cluster Monte Carlo algorithm for lattice models, based on geometric transformations. We prove detailed balance when the transformation is self-inverse, and a symmetry of the Hamiltonian. This algorithm opens new possibilities, in particular for the efficient simulation of critical model systems, where the Metropolis method suffers from critical slowing down. We illustrate the generality of our method by applications to the Ising model in the constant-magnetization ensemble, and to the tricritical Blume-Capel model. [S1063-651X(98)00905-2]

PACS number(s): 05.50.+q, 02.70.Lq, 64.60.Ht
\end{abstract}

The Monte Carlo simulation of a lattice model with a dynamic exponent $z$ and size $L$ in $d$ dimensions requires of the order of $L^{d+z}$ operations to generate an independent configuration. For local Metropolis-type updates at criticality typically $z \approx 2$ : the simulation time increases rapidly with the system size. This "critical slowing down" makes it difficult to explore large critical systems.

This problem was partly solved after a breakthrough due to Swendsen and Wang [1]: the cluster Monte Carlo method. Instead of a single spin, a whole cluster of spins is flipped simultaneously. The construction of these clusters can be formulated as a critical percolation process, so that also clusters of relatively large sizes can be formed. This Monte Carlo process has a nonlocal character, and suppresses critical slowing down.

The Swendsen-Wang algorithm was originally applied to the ferromagnetic $q$-state Potts model, which includes the Ising model. A number of related algorithms has been developed, but their applicability is limited to models that are symmetric under inversion or permutation of spin states.

In this paper, we formulate a cluster algorithm in the context of more general symmetries of the Hamiltonian. In particular, we use geometrical symmetries that map the sites of a lattice model onto one another. To display the role of symmetries, we first recall the Wolff version [2] of the Swendsen-Wang method. We use the language of the spin$\frac{1}{2}$ Ising model with pair interactions $-K s_{i} s_{j}$, but the method described below is more generally applicable. One Wolff step involves the following:

(1) Choose a random lattice site; denote it $i$.

(2) $s_{i}^{\prime}=-s_{i}($ flip spin $i)$.

(3) For all neighbor sites $k$ of $i$ do the following.

(a) If $s_{k}=-s_{i}^{\prime}$ do the following with probability 1 $-e^{-2 K}$ : (i) $s_{k}^{\prime}=-s_{k}$ (spin $k$ included in cluster); (ii) write $k$ in a list of addresses (called stack).

(b) If $s_{k}=s_{i}^{\prime}$, do nothing.

(4) Read an address $j$ from the stack.

(5) Execute the steps listed under (3) for the neighbor sites $k$ of $j$.

(6) Erase the address $j$ from the stack.

(7) Repeat steps (4)-(6) until the stack is empty. When the stack is empty, the Wolff cluster is completed and flipped.
The proof that this process generates the Boltzmann distribution relies on the condition of detailed balance, namely,

$$
T\left(S, S^{\prime}\right) P_{\mathrm{eq}}\left(S^{\prime}\right)=T\left(S^{\prime}, S\right) P_{\mathrm{eq}}(S),
$$

where $T\left(S, S^{\prime}\right)$ denotes the probability of a Wolff step from a spin configuration $S^{\prime}$ to $S$, and $P_{\text {eq }}$ is the Boltzmann distribution. The proof of this condition [2] hinges on a symmetry of the Hamiltonian: the spin inversion symmetry. It also uses the fact that this symmetry is its own inverse. The Wolff algorithm does not work in a field, and for lattice-gas models, where the spin inversion symmetry is absent.

However, there are other symmetries that one may use. Investigating hard-core gases in continuous space, Dress and Krauth [3] developed a cluster method using geometric operations on the particle positions. For hard disks, the percolation threshold of the cluster formation process does not coincide with the phase transition of the model [3]. This is unfortunate since it affects the efficiency of the algorithm.

We applied the idea of using geometric symmetries to lattice models, hoping that the phase transition and the percolation threshold coincide. We may employ reflections, inversions, translations and rotations, provided that these are self-inverse, and global symmetries of the Hamiltonian.

We formulate the geometric cluster formation process here such as to expose the analogy with the Wolff algorithm. Let sites $i, j$, and $k$ map on $i^{\prime}, j^{\prime}$, and $k^{\prime}$ under the symmetry transformation. We denote the energy difference (divided by $k T$ ) when a neighbor $k$ of $i$ is interchanged with $k^{\prime}$ as $\Delta_{i k}$. For the Ising model, $\Delta_{i k}=K\left(s_{i} s_{k}+s_{i^{\prime}} s_{k^{\prime}}-s_{i} s_{k^{\prime}}\right.$ $\left.-s_{i} s_{k}\right)$. The algorithm involves the following steps:

(1) Choose a random lattice site $i ; i$ and $i^{\prime}$ belong to the cluster.

(2) Interchange $s_{i}$ and $s_{i^{\prime}}$.

(3) For all neighbor sites $k$ of $i$ that do not (yet) belong to the cluster, do the following.

(a) If $\Delta_{i k}>0$ do the following with probability 1 $-e^{-\Delta_{i k}}$ : (i) interchange $s_{k}$ and $s_{k^{\prime}}\left(k\right.$ and $k^{\prime}$ included in cluster); (ii) write $k$ in a list of addresses (the stack).

(b) If $\Delta_{i k} \leqslant 0$, do nothing.

(4) Read an address $j$ from the stack.

(5) Execute the steps listed under (3), substituting $j$ for $i$.

(6) Erase the address $j$ from the stack.

(7) Repeat steps (4)-(6) until the stack is empty. 
When the stack is empty, the cluster is completed and moved. Consider the probability $T\left(S^{\prime}, S\right)$ of a cluster move that transforms a spin configuration $S$ into $S^{\prime}$ by moving the spins contained in the geometric cluster $\mathcal{C}$. We verify that the condition of detailed balance,

$$
\frac{T\left(S, S^{\prime}\right)}{T\left(S^{\prime}, S\right)}=\exp \left[\frac{E\left(S^{\prime}\right)-E(S)}{k T}\right]
$$

is satisfied. The energy change $E\left(S^{\prime}\right)-E(S)$ is due to pair interactions between spins inside $\mathcal{C}$ and those outside. It consists of a positive contribution $E_{1}(\mathcal{C}, S)$ due to pair interactions whose energies increase when $S$ changes into $S^{\prime}$, and a negative contribution $-E_{2}(\mathcal{C}, S)$ due to pair interactions whose energies decrease. We write the probability of the cluster move as $T\left(S^{\prime}, S\right)=T_{i}(\mathcal{C}, S) T_{b}(\mathcal{C}, S)$. The "internal" factor $T_{i}$ is the probability that the cluster connects to all sites within $\mathcal{C}$. The "boundary" factor $T_{b}$ is the probability that the cluster does not include spins outside $\mathcal{C} . T_{i}$ depends only on spins in $\mathcal{C}$. According to the above cluster formation rules, $T_{b}(\mathcal{C}, S)=\exp \left[-E_{1}(\mathcal{C}, S) / k T\right]$. The reverse process satisfies $T\left(S, S^{\prime}\right)=T_{i}\left(\mathcal{C}, S^{\prime}\right) T_{b}\left(\mathcal{C}, S^{\prime}\right)$. The first factor equals $T_{i}(\mathcal{C}, S)$ because the operation is self-inverse, and a symmetry of the system. Moreover, $T_{b}\left(\mathcal{C}, S^{\prime}\right)$ $=\exp \left[-E_{1}\left(\mathcal{C}, S^{\prime}\right) / k T\right]=\exp \left[-E_{2}(\mathcal{C}, S) / k T\right]$. Thus

$$
\frac{T\left(S, S^{\prime}\right)}{T\left(S^{\prime}, S\right)}=\exp \left[\frac{E_{1}(\mathcal{C}, S)-E_{2}(\mathcal{C}, S)}{k T}\right] \text {. }
$$

The definitions of $E_{1}$ and $E_{2}$ imply that $E\left(S^{\prime}\right)-E(S)=E_{1}(\mathcal{C}$, $S)-E_{2}(\mathcal{C}, S)$ so that detailed balance, Eq. (2), is indeed satisfied.

These ideas have, in part, already been applied to hardcore lattice gases [4]. For these models the energy change $\Delta_{i k}$ is either zero or infinite, so that the probabilities are 0 or 1 and the cluster formation process becomes deterministic after step 1. It reduces to a special case of the algorithm given above. Histograms of the cluster size distribution, taken at criticality, appeared to scale with $L^{y_{h}}$, where $y_{h}$ is the Ising magnetic exponent. This is the same behavior as for Wolff clusters in the Ising case. Thus, for these lattice gases, the percolation threshold of the geometric clusters corresponds with the phase transition. In line with this observation, critical slowing down is effectively suppressed [4].

However, the presence of finite couplings modifies the cluster formation, and it is not clear that the process remains on the percolation threshold. Thus we applied the algorithm to critical ferromagnetic Ising models in two and three dimensions. The magnetization density was initialized as $m$ $=0$ and is conserved. Using the Wolff scaling factor $L^{y_{h}}$, we again observed that histograms of the geometric cluster size collapse well. Figure 1 shows the result for the twodimensional case.

We proceed by sketching a proof that the cluster sizes scale similarly as in the Wolff case, i.e., the percolation threshold coincides with the phase transition. Fold the Ising model such that $s_{i}$ and $s_{i}$ form adjacent pairs in the ( $d$ $+1)$ th dimension: an Ashkin-Teller model at the decoupling pont. The numbers of such pairs are $N_{++}, N_{+-}, N_{-+}$, and $N_{--}$; the subscripts refer to the signs of $s_{i}$ and $s_{i^{\prime}}$. The

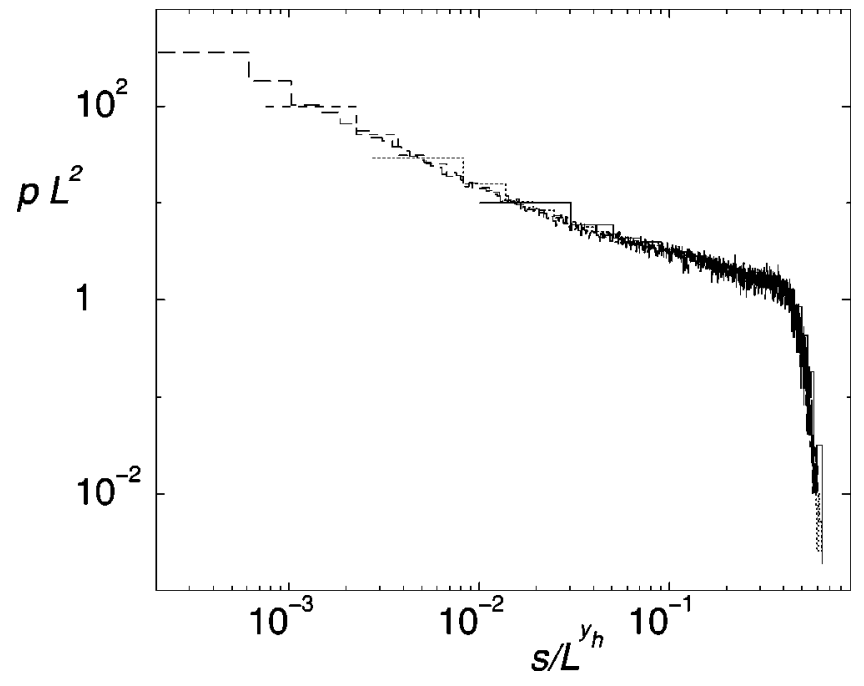

FIG. 1. Scaled histograms of cluster sizes in a simulation at zero magnetization of the critical two-dimensional Ising model. Size $8^{2}$ is shown by a full line, $16^{2}$ by a dotted line, $32^{2}$ by a dashed line, and $64^{2}$ by long dashes.

critical Ising susceptibility $\chi=4 N^{-1}\left\langle\left(N_{++}-N_{--}\right)^{2}\right\rangle$ is proportional to $L^{2 y_{h}-d}$. We neglect the coupling of the sheets containing $s_{i}$ and $s_{i}$, via the boundary conditions. Up-down symmetry then leads to

$$
N^{-1}\left\langle\left(N_{+-}-N_{-+}\right)^{2}\right\rangle \propto L^{2 y_{h}-d} .
$$

Next, divide the Ising lattice into geometric clusters, and ignore those consisting of two equal spins. Each remaining cluster consists of + spins in one sheet and - spins in the other. Its size is denoted $n_{k}(k=1,2, \ldots, M)$, and the sign of its spins in the upper sheet as $p_{k}$. Thus, $N_{+-}-N_{-+}$ $=\Sigma_{k} n_{k} p_{k}$. The signs $p_{k}= \pm 1$ are equally probable, so that

$$
\begin{aligned}
N^{-1} & \left\langle\left(N_{+-}-N_{-+}\right)^{2}\right\rangle \\
& =N^{-1} 2^{-M}\left(\sum_{p_{1}= \pm 1} \cdots \sum_{p_{M}= \pm 1}\left(\sum_{k} n_{k} p_{k}\right)^{2}\right\rangle \\
& =N^{-1}\left\langle\sum_{k} n_{k}^{2}\right\rangle .
\end{aligned}
$$

Since the probability to select a cluster is $n_{k} / N$, Eq. (5) is just the average cluster size produced by the geometric method; as in the Wolff case, it is proportional to $L^{2 y_{h}-d}$. This confirms the critical percolation property of the geometric algorithm, and its power to suppress critical slowing down.

While the efficiency of this algorithm is comparable with the Wolff algorithm, its applicability is different because it samples the constant magnetization ensemble: the magnetic field does not enter the simulation algorithm. An example of a possible application is the determination of the simultaneous probability distribution of the magnetization and the energy, which may be used [5] to find the distribution of Lee-Yang zeros [6] at nonzero $m$, and to calculate thermodynamic observables [7].

As a further test, we calculate the field $h(m)$ from frequencies of "local states" [8], at different magnetizations $m$. 


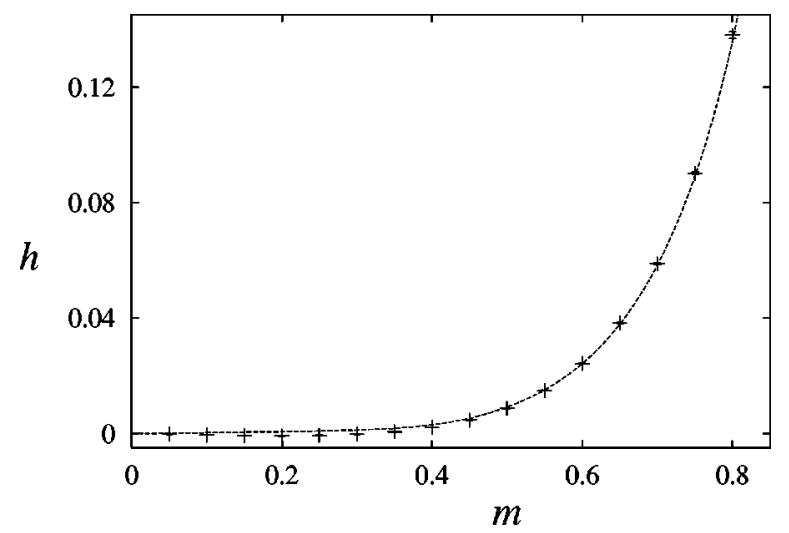

FIG. 2. Magnetic field $h$ as a function of the magnetization density $m$ for a $16^{3}$ Ising model (data points), calculated from local states in cluster simulations at constant magnetization. Also shown is the canonical magnetization as a function of the magnetic field (dashed curve), obtained by conventional Monte Carlo methods.

Results for a $16^{3}$ simple cubic Ising model are shown in Fig. 2 . For comparison, the canonical relation $m(h)$ was obtained from reweighted Wolff (for small $h \leqslant 0.01$ ) and Metropolis simulations (for $\geqslant 0.01$ ). The agreement is good at large $m$; for small $m$, fluctuations are important and differences are visible.

Finally, the geometric cluster method was applied to the Blume-Capel model on the simple cubic lattice. The spins assume the values \pm 1 and 0 ; an additional energy $D k T$ is assigned to each nonzero spin:

$$
\mathcal{H} / k T=-K \sum_{\langle i, j\rangle} s_{i} s_{j}+D \sum_{k} s_{k}^{2}
$$

This model can be simulated by combined Wolff and Metropolis steps [9]. However, for larger $D$ a tricritical point occurs where large fluctuations occur in the number of vacancies (spins with value 0), and critical slowing down reappears.

Preliminary work [10] locates this tricritical point near $K=0.7194, \quad D=2.05$, where the density of the vacancies is $\rho_{\mathrm{vac}} \approx 0.61$. In addition to $K$, we used the density of the vacancies (and not the conjugate parameter $D$ ) in order to fix the position of the system in the phase diagram. After initialization, $\rho_{\text {vac }}=0.61$ remains constant, just as the magnetization that was chosen remains $m=0$. The value of $D$ may be determined from local states in analogy with $h(m)$. The av-

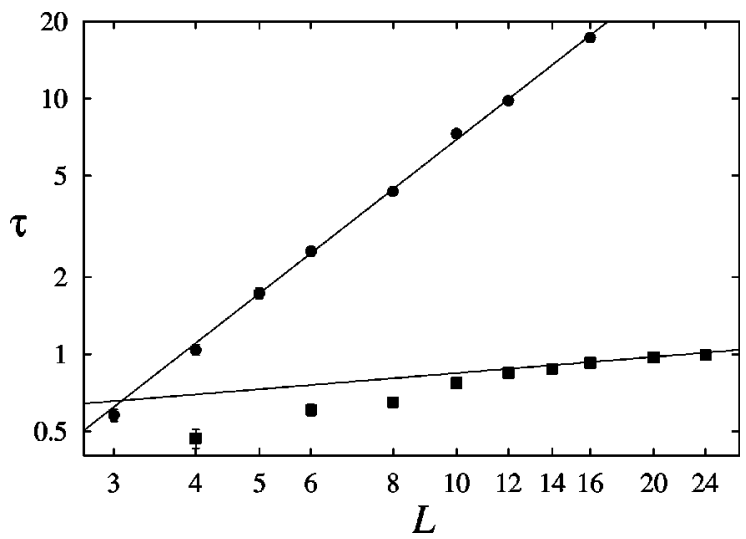

FIG. 3. Autocorrelation times $\tau$ of the energy (nearest-neighbor sum) for single-spin updates (circles) and for the geometric cluster method (squares) vs system size, using logarithmic scales. The straight lines correspond with dynamic exponents $z=2.2$ and with $z=0.21$.

erage cluster size appears to grow algebraically with $L$, which indicates that again the process occurs near the percolation threshold.

Figure 3 displays energy relaxation times at the estimated tricritical point, for single spin updates as well as for the new cluster method. In both cases we chose the time unit as the number of steps needed to update or move $L^{3}$ spins. Thus, the "work" per unit of time is of the same order. For single spin updates we find a dynamic exponent of about $z=2.2$. The cluster method yields an effective exponent of $z=0.21$ in the range $12<L \leqslant 24$ : again, critical slowing down is small. The result $z=0.21$ seems to violate the Li-Sokal [11] bound $z \geqslant \alpha / \nu=1$ (for $\alpha=\nu=0.5$ ). However, the conservation of $\rho_{\text {vac }}$ suppresses energy fluctuations and the canonical tricritical value $\alpha=0.5$ does not apply.

In conclusion, our results show that the geometric cluster algorithm effectively suppresses critical slowing down, at least for a number of Isinglike models. It opens new possibilities for applications to models that were, until now, outside the reach of cluster simulations. Furthermore, one may investigate different ensembles, for instance, the constant magnetization ensemble in the case of the Ising model. While the efficiency of new applications to critical systems still depends on the question of whether the geometric clusters are formed at the percolation threshold, the results presented here are promising.

H.B. is indebted to Professor J.M.J. van Leeuwen for his elucidating comments.
[1] R. H. Swendsen and J.-S. Wang, Phys. Rev. Lett. 58, 86 (1987).

[2] U. Wolff, Phys. Rev. Lett. 62, 361 (1989).

[3] C. Dress and W. Krauth, J. Phys. A 28, L597 (1995).

[4] J. R. Heringa and H. W. J. Blöte, Physica A 232, 369 (1996); Physica A 251, 224 (1998).

[5] G. Bhanot, R. Salvador, S. Black, P. Carter, and R. Toral, Phys. Rev. Lett. 59, 803 (1987).
[6] T. D. Lee and C. N. Yang, Phys. Rev. 87, 410 (1952).

[7] K.-C. Lee, J. Phys. A 23, 2087 (1990).

[8] K. Binder, Rep. Prog. Phys. 60, 487 (1997).

[9] H. W. J. Blöte, E. Luijten, and J. R. Heringa, J. Phys. A 28, 6289 (1995).

[10] H. W. J. Blöte and J. R. Heringa (unpublished).

[11] X.-J. Li and A. D. Sokal, Phys. Rev. Lett. 63, 827 (1989). 